\title{
An Iterative Receiver for Quantized MIMO Systems
}

\author{
Amine Mezghani, Mehdi Rouatbi and Josef A. Nossek \\ Institute for Circuit Theory and Signal Processing \\ Munich University of Technology, 80290 Munich, Germany \\ E-Mail: \{Mezghani, Nossek\}@nws.ei.tum.de
}

\begin{abstract}
We study the joint optimization of the quantizer and the iterative Decision Feedback Equalizer (IDE) for the flat multiinput multi-output (MIMO) channel with quantized outputs. Our design is based on a minimum mean square error (MMSE) approach, taking into account the effects of quantization. Our derivation does not make use of the assumption of uncorrelated white quantization errors and considers the correlations of the quantization error with the other signals of the system. Through simulation, we compare the provided IDE to the conventional spatial DFE operating on quantized data in terms of uncoded BER.
\end{abstract}

\section{INTRODUCTION}

Multi-input multi-output (MIMO) technology constitutes a breakthrough in the design of wireless communication systems, and is already at the core of several wireless standards. A considerable research effort is being spent towards performance enhancements in term of data transmission rate and interference reduction, as well as the reduction of receiver complexity in MIMO systems.

However, most of the contributions on receiver design for MIMO systems assumes that the receiver has access to the channel data with infinite precision. In practice, tough, a quantizer is applied to the received signal, which limits the accuracy of continuous valued channel measurements, so that they can be proceeded in the digital domain. So far, the effect of the quantization has been neglected in most of the research work due to the complexity of the theoretical analysis, and since signal quantization is a non-linear operation. Furthermore, there exist rather high-resolution analog-to-digital converters (ADC) which are believed to produce a quantized signal of such a fine granularity, that its effects can either be neglected completely or taken into account by a linear model considering the quantization error as an additive noise, which is nearly uncorrelated (or even nearly independent) with the received signal [1]. However, the reliance on fine analog-to-digital converters (ADCs) granularity easily becomes unjustified as soon as we have to do with high-speed MIMO channels [2]. In this case, the needed ADCs to fulfill this assumption are expensive and even no more feasible. In fact, in order to reduce circuit complexity and save power and area, low resolution ADCs have to be employed [3]. Therefore, the proposed receiver designs do not necessarily have good performance when operating on quantized data in a real system. In [2] and [4], the effects of quantization are studied from an information theoretical point of view. In [5], the authors examined these effects experimentally by using a standard Zero-Forcing filter at the receiver.

In this work, we focus on the receive signal processing for quantized MIMO systems. The receive equalizers will be then designed upon the knowledge of the Channel State Information
(CSI) and the a priori defined transmit filter (in the simple case, the transmit matrix is equal to the identity matrix). Linear equalization techniques used for MIMO-receivers design are only optimum in some special cases and can be outperformed by nonlinear receivers in term of bit error ratio, and are therefore not efficient in some wireless applications. The optimum non-linear technique is the Maximum Likelihood (ML) detection, that has an exponential complexity [6] which is prohibitive in general, and hence becomes quickly inconvenient to high signal constellations and high numbers of transmit antennas. Among non-linear equalization techniques, Decision Feedback Equalization (DFE) is the most common suboptimum solution, since it is fairly simple to implement and generally performs well. The Iterative Decision Equalization (IDE) [7] has been also developed to mitigate this complexity problem, and it is appropriate for large systems (high number of antennas). The IDE detectors cancel iteratively the interference from the received signal and generate symbol decisions whose reliability increases monotonically with each iteration. The concept of combining the iterative equalization with decoding technique for coded data transmission (Turbo Equalization), can achieve impressive performance gains in communication systems. The main contribution of this paper is the application of this technique for quantized MIMO systems while taking the effects of quantization in a systematic way. Our paper is organized as follows. First, we introduce the system model and some notational issues in section II. In section III, we discuss the properties of the chosen quantizer, then we present the modified IDE receiver in section IV. Finally, we present some simulation results in section V.

\section{System Model And Notation}

We consider a point-to-point MIMO Gaussian channel where the transmitter employs $M$ antennas and the receiver has $N$ antennas. Fig. 1 shows the general form of a quantized MIMO system, where $\boldsymbol{H} \in \mathbb{C}^{N \times M}$ is the channel matrix. The vector $\boldsymbol{x} \in \mathbb{C}^{M}$ comprises the $M$ transmitted symbols, which are uncorrelated and have zero-mean and covariance matrix $\boldsymbol{R}_{x x}=\mathrm{E}\left[\boldsymbol{x} \boldsymbol{x}^{\mathrm{H}}\right]=\sigma_{x}^{2} \mathbf{I}$. The vector $\boldsymbol{\eta}$ refers to zeromean complex circular Gaussian noise with covariance $\boldsymbol{R}_{\eta \eta}=$ $\mathrm{E}\left[\boldsymbol{\eta} \boldsymbol{\eta}^{\mathrm{H}}\right] . \boldsymbol{y} \in \mathbb{C}^{N}$ is the unquantized channel output:

$$
\boldsymbol{y}=\boldsymbol{H} \boldsymbol{x}+\boldsymbol{\eta} .
$$

In our system, the real parts $y_{i, R}$ and the imaginary parts $y_{i, I}$ of the receive signals $y_{i}, 1 \leq i \leq N$, are each quantized by a $b$-bit resolution uniform/non-uniform scalar quantizer. Thus, the resulting quantized signals read as:

$$
r_{i, l}=Q\left(y_{i, l}\right)=y_{i, l}+q_{i, l}, l \in\{R, I\}, 1 \leq i \leq N,
$$

where $Q(\cdot)$ denotes the quantization operation and $q_{i, l}$ is the resulting quantization error.

In the IDE structure shown in Fig. 1, the received quantized 
vector $r$ is multiplied at the $l$-th iteration by the forward filter matrix $\boldsymbol{G}^{(l)} \in \mathbb{C}^{M \times N}$

$$
\tilde{\boldsymbol{r}}^{(l)}=\boldsymbol{G}^{(l)} \boldsymbol{r}
$$

then rectified from the constructed estimate $\boldsymbol{F}^{(l)} \hat{\boldsymbol{x}}^{(l-1)}$ of the interference at the previous iteration $(l-1)$, producing the $M$-dimensional vector

$$
\tilde{\boldsymbol{x}}^{(l)}=\boldsymbol{G}^{(l)} \boldsymbol{r}-\boldsymbol{F}^{(l)} \hat{\boldsymbol{x}}^{(l-1)},
$$

or , equivalently,

$$
\tilde{x}_{k}^{(l)}=\boldsymbol{g}_{k}^{(l), \mathrm{T}} \boldsymbol{r}-\boldsymbol{f}_{k}^{(l), \mathrm{T}} \hat{\boldsymbol{x}}^{(l-1)}, \quad \forall k=1,2, \ldots, M .
$$

Our aim is to choose the quantizer, the receive matrix $\boldsymbol{G}$, and the feedback matrix $\boldsymbol{F}$, minimizing the MSE $=\mathrm{E}\left[\|\hat{\boldsymbol{x}}-\boldsymbol{x}\|_{2}^{2}\right]$, taking into account the quantization effect. Throughout this paper, $r_{\alpha \beta}$ denotes $\mathrm{E}\left[\alpha \beta^{*}\right]$. The operators $(\bullet)^{\mathrm{T}},(\bullet)^{\mathrm{H}},(\bullet)^{*}$, $\operatorname{Re}(\bullet), \operatorname{Im}(\bullet)$ stand for transpose, Hermitian transpose, complex conjugate, real and imaginary parts of a complex number, respectively.

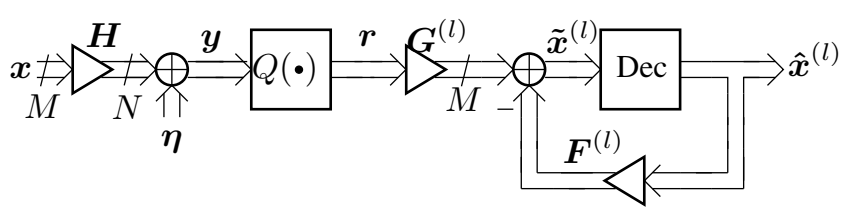

Fig. 1. Iterative Decision Equalizer on a quantized MIMO Channel.

\section{QUANTIZER DESCRIPTION}

Each quantization process is given a distortion factor $\rho_{q}^{(i, l)}$ to indicate the relative amount of quantization noise generated, which is defined as follows

$$
\rho_{q}^{(i, l)}=\frac{\mathrm{E}\left[q_{i, l}^{2}\right]}{r_{y_{i, l} y_{i, l}}}
$$

where $r_{y_{i, l} y_{i, l}}=\mathrm{E}\left[y_{i, l}^{2}\right]$ is the variance of $y_{i, l}$ and the distortion factor $\rho_{q}^{(i, l)}$ depends on the number of quantization bits $b$, the quantizer type (uniform or non-uniform) and the probability density function of $y_{i, l}$. Note that the signal-to-quantization noise ratio (SQNR) has an inverse relationship with regard to the distortion factor

$$
\operatorname{SQNR}^{(i, l)}=\frac{1}{\rho_{q}^{(i, l)}}
$$

Similar to our work [8] concerning the Wiener filter for quantized data, the uniform/non-uniform quantizer design is based on minimizing the mean square error (distortion) between the input $y_{i, l}$ and the output $r_{i, l}$ of each quantizer. In other words, the SQNR values are maximized. Under this optimal design of the scalar finite resolution quantizer, whether uniform or not, the following equations hold for all $0 \leq i \leq N, l \in\{R, I\}$ [9], [10]:

$$
\begin{aligned}
\mathrm{E}\left[q_{i, l}\right] & =0 \\
\mathrm{E}\left[r_{i, l} q_{i, l}\right] & =0 \\
\mathrm{E}\left[y_{i, l} q_{i, l}\right] & =-\rho_{q}^{(i, l)} r_{y_{i, l} y_{i, l}} .
\end{aligned}
$$

Obviously, Eq. (10) follows from Eqs (6) and (9). For the uniform quantizer case, Eq. (8) holds only if the probability density function of $y_{i, l}$ is even.
Under multipath propagation conditions and for large number of antennas, the quantizer input signals $y_{i, l}$ are approximately Gaussian distributed and thus, they undergo nearly the same distortion factor $\rho_{q}$, i.e., $\rho_{q}^{(i, l)}=\rho_{q} \forall i \forall l$. Furthermore, the optimal parameters of the uniform as well as the nonuniform quantizer and the resulting distortion factor $\rho_{q}$ for Gaussian distributed signal are tabulated in [9] for different bit resolutions $b$. Recent research work on optimally quantizing the Gaussian source can be found in [11].

Now, let $q_{i}=q_{i, R}+\mathrm{j} q_{i, I}$ be the complex quantization error. Under the assumption of uncorrelated real and imaginary part of $y_{i}$, we easily obtain:

$r_{q_{i} q_{i}}=\mathrm{E}\left[q_{i} q_{i}^{*}\right]=\rho_{q} r_{y_{i} y_{i}}$, and $r_{y_{i} q_{i}}=\mathrm{E}\left[y_{i} q_{i}^{*}\right]=-\rho_{q} r_{y_{i} y_{i}}$.

For the uniform quantizer case, it was shown in [11], that the optimal quantization step $\Delta$ for a Gaussian source decreases as $\sqrt{b} 2^{-b}$ and that $\rho_{q}$ is asymptotically well approximated by $\frac{\Delta^{2}}{12}$ and decreases as $b 2^{-2 b}$. On the other hand, the optimal nonuniform quantizer achieves, under high-resolution assumption, approximately the following distortion [12]

$$
\rho_{q} \approx \frac{\pi \sqrt{3}}{2} 2^{-2 b}
$$

This particular choice of the (non-)uniform scalar quantizer minimizing the distortion between $\boldsymbol{r}$ and $\boldsymbol{y}$, combined with the receiver of the next section turns to be optimal with respect to the total MSE between the transmitted symbol vector $\boldsymbol{x}$ and the estimated symbol vector $\tilde{\boldsymbol{x}}^{(l)}$ at each iteration $l$.

\section{NEAR OPTIMAL IDE RECEIVER FOR THE QUANTIZED SYSTEM}

Among the possible criteria to optimize the receive filter $\boldsymbol{G}^{(l)}$ and the feedback matrix $\boldsymbol{F}^{(l)}$ for the IDE detection on quantized data, we consider here the minimization of the MSE between the sent vector $\boldsymbol{x}$ and the estimated vector $\tilde{\boldsymbol{x}}^{(l)}$, taking into account the reliability of tentative decisions as well as the effect of quantization.

The MSE minimization is extended by an unbiasedness constraint to avoid direct feedback of the detected symbols, so that from each input symbol $\tilde{x}_{k}^{(l)}$ at the decision module only the interference, caused by others symbols $\hat{x}_{j}^{(l-1)}$ for $j \neq k$, is subtracted. In this case, the MSE minimization can be mathematically expressed as

$$
\min _{\left\{\boldsymbol{G}^{(l)}, \boldsymbol{F}^{(l)}\right\}} \mathrm{E}\left[\left\|\tilde{\boldsymbol{x}}^{(l)}-\boldsymbol{x}\right\|_{2}^{2}\right] \quad \text { s.t. }: \boldsymbol{g}_{k}^{(l), \mathrm{T}} \boldsymbol{h}_{k}=\frac{1}{1-\rho_{q}} .
$$

The MSE between each sent symbol $x_{k}$ and its estimated symbol $\tilde{x}_{k}^{(l)}(1 \leq k \leq M)$ reads as

$$
\operatorname{MSE}_{\mathrm{IDEQ}, k}^{(l)}=\varepsilon_{k}^{(l)}=\mathrm{E}\left[\left\|\tilde{x}_{k}^{(l)}-x_{k}\right\|_{2}^{2}\right],
$$

where,

$$
\tilde{x}_{k}^{(l)}-x_{k}=\boldsymbol{g}_{k}^{(l), \mathrm{T}} \boldsymbol{\eta}+\boldsymbol{g}_{k}^{(l), \mathrm{T}} \boldsymbol{q}+\left(\boldsymbol{g}_{k}^{(l), \mathrm{T}} \boldsymbol{H}-\boldsymbol{e}_{k}^{\mathrm{T}}\right) \boldsymbol{x}-\boldsymbol{f}_{k}^{(l), \mathrm{T}} \hat{\boldsymbol{x}}^{(l-1)},
$$

with $\boldsymbol{e}_{k}^{\mathrm{T}}$ being the $1 \times M$ row vector that is zero except for the $k$-th element, which is one, and $\boldsymbol{q}$ denotes the quantization error vector. The calculation of the MSE needs the correlation matrix

$$
\boldsymbol{R}_{x r}=\mathrm{E}\left[\boldsymbol{x} \boldsymbol{r}^{\mathrm{H}}\right]=\mathrm{E}\left[\boldsymbol{x}(\boldsymbol{y}+\boldsymbol{q})^{\mathrm{H}}\right]=\boldsymbol{R}_{x y}+\boldsymbol{R}_{x q},
$$


and the covariance matrix of the quantized signal given by

$$
\boldsymbol{R}_{r r}=\mathrm{E}\left[(\boldsymbol{y}+\boldsymbol{q})(\boldsymbol{y}+\boldsymbol{q})^{\mathrm{H}}\right]=\boldsymbol{R}_{y y}+\boldsymbol{R}_{y q}+\boldsymbol{R}_{y q}^{\mathrm{H}}+\boldsymbol{R}_{q q}
$$

as well as the correlation matrix between the exact input vector $\boldsymbol{x}$ and its reconstructed value $\hat{\boldsymbol{x}}^{(l-1)}$ at step $l-1$

$$
\frac{\mathrm{E}\left[\boldsymbol{x} \cdot \hat{\boldsymbol{x}}^{(l-1), \mathrm{H}}\right]}{\sigma_{x}^{2}}=\boldsymbol{\rho}^{(l-1)}=\operatorname{diag}\left(\rho_{1}^{(l-1)}, \rho_{2}^{(l-1)}, \ldots, \rho_{M}^{(l-1)}\right),
$$

where $\rho_{k}^{(l-1)}$ can be interpreted as a measure of the reliability of $\hat{x}_{k}^{(l-1)}$. More generally, $\boldsymbol{\rho}^{(l-1)}$ describes our confidence in the quality of the estimate $\hat{\boldsymbol{x}}^{(l-1)}$. If $\hat{\boldsymbol{x}}^{(l-1)}$ is a poor estimate of $\boldsymbol{x}$, then $\boldsymbol{\rho}^{(l-1)}$ correspondingly be close to zero, and consequently a smaller weighting is applied to the interference estimate that is to be subtracted from $\tilde{\boldsymbol{r}}^{(l)}$. Otherwise, if $\hat{\boldsymbol{x}}^{(l-1)}$ is an excellent estimate of $\boldsymbol{x}$, then $\boldsymbol{\rho}^{(l-1)} \approx \boldsymbol{I}$, and almost the complete interference is removed from $\tilde{\boldsymbol{r}}^{(l)}$. For square $L$ QAM modulation, we use the following approximation based on the nearest neighbor error assumption

$$
\rho_{k}^{(l)} \approx 1-\frac{3}{L-1} \operatorname{Pr}(\text { Error }),
$$

with

$$
\operatorname{Pr}(\text { Error }) \approx 1-\left(1-2\left(1-\frac{1}{\sqrt{L}}\right) \mathcal{Q}\left(\sqrt{\frac{3 \sigma_{x}^{2}}{(L-1) \epsilon_{k}^{(l)}}}\right)\right)^{2} .
$$

\section{A. Derivation of the DFEQ Receiver}

Before investigating the MMSE optimization, we first derive all needed covariance matrices by using the fact that the quantization error $q_{i}$, conditioned on $y_{i}$, is statistically independent from all other random variables of the system.

First we calculate $r_{y_{i} q_{j}}=\mathrm{E}\left[y_{i} q_{j}^{*}\right]$ for $i \neq j$ :

$$
\begin{aligned}
\mathrm{E}\left[y_{i} q_{j}^{*}\right] & =\mathrm{E}_{y_{j}}\left[\mathrm{E}\left[y_{i} q_{j}^{*} \mid y_{j}\right]\right] \\
& =\mathrm{E}_{y_{j}}\left[\mathrm{E}\left[y_{i} \mid y_{j}\right] \mathrm{E}\left[q_{j}^{*} \mid y_{j}\right]\right] \\
& \approx \mathrm{E}_{y_{j}}\left[r_{y_{i} y_{j}} r_{y_{j} y_{j}}^{-1} y_{j} \mathrm{E}\left[q_{j}^{*} \mid y_{j}\right]\right] \\
& =r_{y_{i} y_{j}} r_{y_{j} y_{j}}^{-1} \mathrm{E}\left[y_{j} q_{j}^{*}\right] \\
& =-\rho_{q} r_{y_{i} y_{j}} .
\end{aligned}
$$

In (21), we approximate the Bayesian estimator $\mathrm{E}\left[y_{i} \mid y_{j}\right]$ with the linear estimator $r_{y_{i} y_{j}} r_{y_{j} y_{j}}^{-1} y_{j}$, which holds with equality if the vector $\boldsymbol{y}$ is jointly Gaussian distributed. Eq. (22) follows from (11). Summarizing the results of (11) and (22), we obtain:

$$
\boldsymbol{R}_{y q} \approx-\rho_{q} \boldsymbol{R}_{y y} .
$$

Similarly, we evaluate $r_{q_{i} q_{j}}$ for $i \neq j$ to end up in:

$$
\mathrm{E}\left[q_{i} q_{j}^{*}\right]=\mathrm{E}_{y_{j}}\left[\mathrm{E}\left[q_{i} q_{j}^{*} \mid y_{j}\right]\right] \approx \rho_{q}^{2} r_{y_{j} y_{i}}^{*}=\rho_{q}^{2} r_{y_{i} y_{j}},
$$

where we used Eqs (23) and (11). From (24) and (11) we deduce the covariance matrix of the quantization error:

$$
\begin{aligned}
\boldsymbol{R}_{q q} & \approx \rho_{q} \operatorname{diag}\left(\boldsymbol{R}_{y y}\right)+\rho_{q}^{2} \operatorname{nondiag}\left(\boldsymbol{R}_{y y}\right) \\
& =\rho_{q} \boldsymbol{R}_{y y}-\left(1-\rho_{q}\right) \rho_{q} \operatorname{nondiag}\left(\boldsymbol{R}_{y y}\right),
\end{aligned}
$$

with $\operatorname{diag}(\boldsymbol{A})$ denotes a diagonal matrix containing only the diagonal elements of $\boldsymbol{A}$ and nondiag $(\boldsymbol{A})=\boldsymbol{A}-\operatorname{diag}(\boldsymbol{A})$. Inserting the expressions (23) and (25) into Eq. (17), we obtain:

$$
\boldsymbol{R}_{r r} \approx\left(1-\rho_{q}\right)\left(\boldsymbol{R}_{y y}-\rho_{q} \operatorname{nondiag}\left(\boldsymbol{R}_{y y}\right)\right)
$$

In a very similar way, we get the covariance matrix $\boldsymbol{R}_{x q}=$ $\mathrm{E}\left[\boldsymbol{x} \boldsymbol{q}^{\mathrm{H}}\right]$ as

$$
\mathrm{E}\left[\boldsymbol{x} \boldsymbol{q}^{\mathrm{H}}\right] \approx-\rho_{q} \boldsymbol{R}_{x y}
$$

Thus, Equation (16) becomes

$$
\boldsymbol{R}_{x r} \approx\left(1-\rho_{q}\right) \boldsymbol{R}_{x y} .
$$

Finally, $\boldsymbol{R}_{y y}$ and $\boldsymbol{R}_{x y}$ can be easily obtained from our system model:

$$
\begin{aligned}
& \boldsymbol{R}_{y y}=\boldsymbol{R}_{\eta \eta}+\sigma_{x}^{2} \boldsymbol{H} \boldsymbol{H}^{\mathrm{H}}, \\
& \boldsymbol{R}_{x y}=\sigma_{x}^{2} \boldsymbol{H}^{\mathrm{H}} .
\end{aligned}
$$

Having calculated the correlation matrices, we use the Lagrangian multiplier method to solve the MSE minimization problem. We omit the derivation and we provide the following final solution for the filters

$$
\boldsymbol{g}_{k}^{(l), \mathrm{T}}=\frac{1}{1-\rho_{q}} \alpha_{k}^{(l)} \boldsymbol{h}_{k}^{\mathrm{H}} \boldsymbol{T}^{(l)},
$$

with

$\boldsymbol{T}^{(l)}=\left[\sigma_{\eta}^{2} \boldsymbol{I}+\sigma_{x}^{2} \boldsymbol{H}\left(\boldsymbol{I}-\boldsymbol{\rho}^{(l-1)} \boldsymbol{\rho}^{(l-1), \mathrm{H}}\right) \boldsymbol{H}^{\mathrm{H}}\right]^{-1} \in \mathbb{C}^{N \times N}$.

and

$$
\alpha_{k}^{(l)}=\frac{1}{\boldsymbol{h}_{k}^{\mathrm{H}} \boldsymbol{T}^{(l)} \boldsymbol{h}_{k}} .
$$

Therefore, the optimal receive filter $G^{(l)}$ for the IDE detection on an unquantized MIMO system reads as

$$
\boldsymbol{G}_{\mathrm{IDEQ}}^{(l)}=\frac{1}{1-\rho_{q}} \boldsymbol{\Theta}^{(l)} \boldsymbol{H}^{\mathrm{H}} \boldsymbol{T}^{(l)},
$$

where $\boldsymbol{\Theta}^{(l)}$ is a diagonal matrix with the elements $\alpha_{k}^{(l)}$. On the other hand, the resulting optimal feedback matrix is obtained as:

$$
\boldsymbol{F}_{\mathrm{IDEQ}}^{(l)}=\left(\boldsymbol{\Theta}^{(l)} \boldsymbol{H}^{\mathrm{H}} \boldsymbol{T}^{(l)} \boldsymbol{H}-\boldsymbol{I}\right) \boldsymbol{\rho}^{(l-1)} .
$$

We notice that the diagonal elements of the feedback matrix are equal to zero $\left[\boldsymbol{F}_{\mathrm{IDE}}^{(l)}\right]_{k k}=0$, so that we avoid direct feedback of the detected symbols as we have mentioned before.

Some comments can be made about the special case when $l=1$. During the first pass, the feedback loop is not used because $\boldsymbol{\rho}^{(0)}=\mathbf{0}$, so the vector $\hat{\boldsymbol{x}}^{(0)}$ does not need to be defined, and is never required for the first iteration. Moreover, the filter $\boldsymbol{G}_{\mathrm{IDEQ}}^{(1)}$ takes the form

$$
\boldsymbol{G}_{\mathrm{IDEQ}}^{(1)}=\frac{1}{1-\rho_{q}} \boldsymbol{\Theta}^{(1)} \boldsymbol{H}^{\mathrm{H}}\left[\boldsymbol{R}_{y y}-\rho_{q} \operatorname{nondiag}\left(\boldsymbol{R}_{y y}\right)\right]^{-1},
$$

which is an expression for the unbiased linear MMSE receiver (Wiener Filter) with quantized channel output derived in [8]. Thus the performance of the iterative decision receiver, after just one iteration, is identical to the performance of the linear MMSE receiver for quantized systems. However, with more iterations, we show later that the iterative decision detector performs significantly better than the linear MMSE detector. 


\section{Simulation Results}

We consider a Rayleigh fading channel, where the entries of $\boldsymbol{H}$ are complex-valued realizations of independent zero-mean Gaussian random variables. For each channel realization, 100 QPSK modulated symbols are transmitted. The simulated bit error ratio (BER) is averaged over $10^{5}$ channels realizations. The noise covariance matrix is $\boldsymbol{R}_{\eta \eta}=\sigma_{\eta}^{2} \mathbf{I}$.

In Fig. 2, the uncoded-BER is plotted as a function of the SNR for 1, 2, 3, 4, 5 and 10 iterations. We choose a MIMO system where the transmitter employs $M=10$ antennas and the receiver $N=12$ antennas. We see clearly that, few passes are required to converge to the target BER. We emphasize that the performance of the first iteration is that of an unbiased linear MMSE receiver for quantized MIMO system (WFQ) [8], so we obtain with more iterations, significantly better performance than the linear WFQ detector.

In Fig. 3, we compare the uncoded-BER of the proposed IDE-detection for a 3- and 4-bit quantized MIMO system (IDEQ) to the IDE ignoring the quantization process [7], the decision feedback filter (DFEQ) [13] and the Wiener filter (WFQ) [8]. We take again a MIMO system where the transmitter employs $M=10$ antennas and the receiver $N=12$ antennas. First of all, we see clearly an improvement in the achieved BER with the IDE-detection compared to the DFE and WF at moderate SNR. This means that, similarly to the unquantized case, the blockwise interference suppression becomes more advantageous than successive interference cancellation for a higher number of antennas. On the other hand, the modified IDE-detection (IDEQ) outperforms clearly the IDE-detection that not considers the quantization at high SNR, where the quantization error is more important than the additive Gaussian noise. Thus, the consideration of the quantization effect enables better performance and improves the quality of the Iterative Decision Equalization at high SNR.

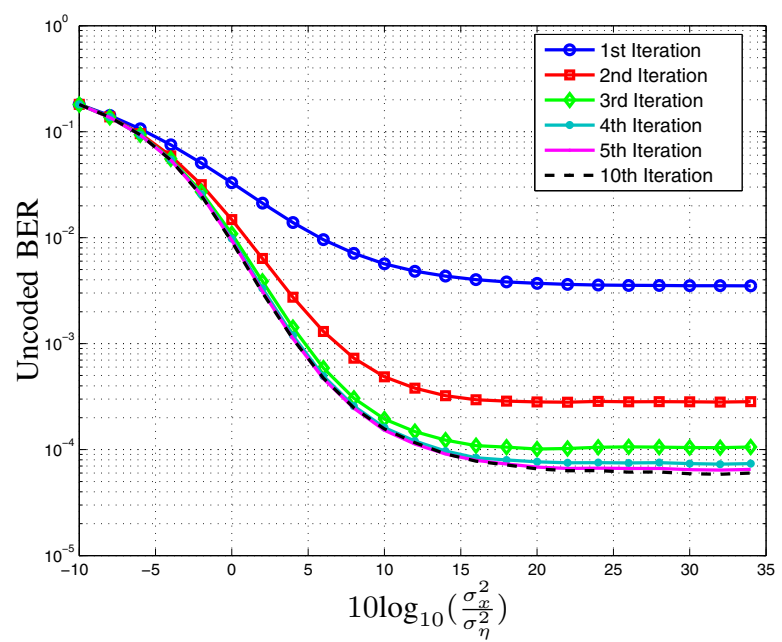

Fig. 2. IDE detector performance (BER) as a function of SNR for 1, 2, 3, 4, 5 and 10 decoding iterations, QPSK modulation with $M=10$ and $N=12$, 3- $\left(\rho_{q}=0.037411\right)$ bit uniform quantizer.

\section{CONCLUSION}

We addressed the problem of designing an iterative decision feedback receiver for MIMO channels with quantized outputs.

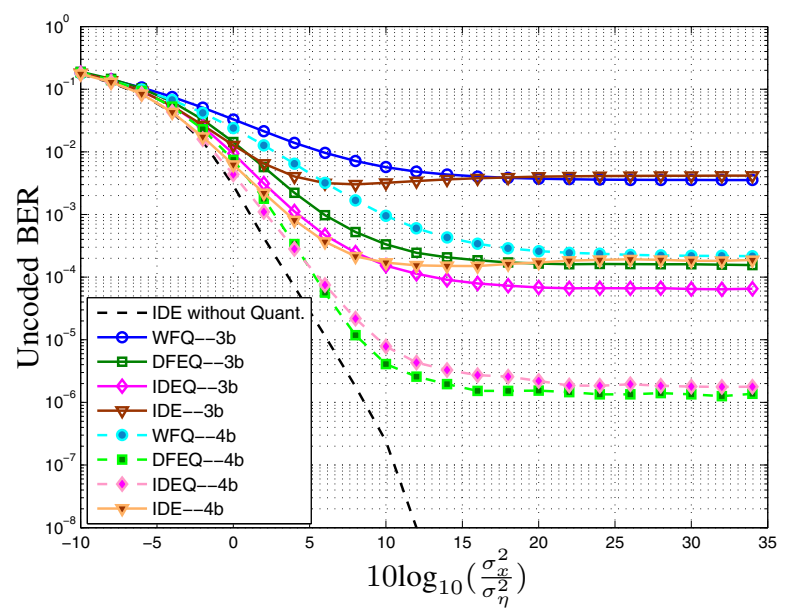

Fig. 3. IDEQ vs. IDE detection (5-th iteration), DFEQ and WFQ receivers, QPSK modulation with $M=10$ and $N=12,3-\left(\rho_{q}=0.037411\right)$ and $4-\left(\rho_{q}=0.01154\right)$ bit uniform quantizer.

We provided an approximation of the correlation matrices needed for evaluating the mean squared error, where the quantizer is optimized for a Gaussian input. Then, we proposed an optimized IDE receiver operating on quantized data, which shows better performance in terms of BER than the previously designed filter especially for large number of antennas. An essential aspect of our derivation is that we do not make the assumption of uncorrelated white quantization error. Moreover, our receiver does not present much extra complexity from the implementation point of view.

\section{REFERENCES}

[1] A. V. Oppenheim and R. W. Schafer, Discrete Time Signal Processing, Prentice Hall, April 1989.

[2] M. T. Ivrlač and J. A. Nossek, "Capacity and Coding-Performance of Quantized MIMO Systems," IEEE International Wireless Communications and Mobile Computing Conference, IWCMC06, (in press), Vancouver, Canada, 2006.

[3] R. Schreier and G. C. Temes, Understanding Delta-Sigma Data Converters, IEEE Computer Society Press, December 2004.

[4] M. T. Ivrlač and J. A. Nossek, "Challenges in Coding for Quantized MIMO Systems," IEEE International Symposium on Information Theory, Seattle, U.S.A., pp. 2114-2118, July 2006.

[5] ] B. Murray and I. B. Collings, "AGC and Quantization Effects in a Zero- Forcing MIMO Wireless System," Proc. of the IEEE Int. Vehicular Technology Conf. (VTC) - Spring, Melbourne, Australia, May 2006.

[6] Jr. Forney, G., "Maximum-Likelihood Sequence Estimation of Digital Sequences in the Presence of Intersymbol Interference," IEEE Transactions on Information Theory, Issue 3, vol. 18, no. 3, pp. 363 - 378, May 1972.

[7] Albert M. Chan, "A Framework for Low Complexity Iterative Interference Cancellation in Communication Systems," Tech. Rep., Massachusetts Institue of Technology, Department of Electrical Engineering and Computer Science, June 2004.

[8] A. Mezghani, M. S. Khoufi, and J. A. Nossek, "A Modified MMSE Receiver for Quantized MIMO Systems," In Proc. ITG/IEEE WSA, Vienna, Austria, February 2007.

[9] J. Max, "Quantization for Minimum Distortion," IEEE Trans. Inf. Theory, vol. 6, no. 1, pp. 7-12, March 1960.

[10] J.G. Proakis, Digital Communications, McGraw Hill, New York, third edition, 1995

[11] Dennis Hui and David L. Neuhoff, "Asymptotic Analysis of Optimal Fixed-Rate Uniform Scalar Quantization," IEEE Trans. Inform. Theory, vol. 47, no. 3, pp. 957-977, March 2001.

[12] A. Gersho and R. M. Gray, Vector Quantization and Signal Compression, Kluwer Academic Publishers, Dordrecht, Niederlande, first edition, 1992.

[13] A. Mezghani, M. S. Khoufi, and J. A. Nossek, "Spatial MIMO Decision Feedback Equalizer Operating on Quantized Data," In Proc. IEEE Int. Conf. Acoust.,Speech, Signal Processing, Las Vegas, USA, April 2008. 